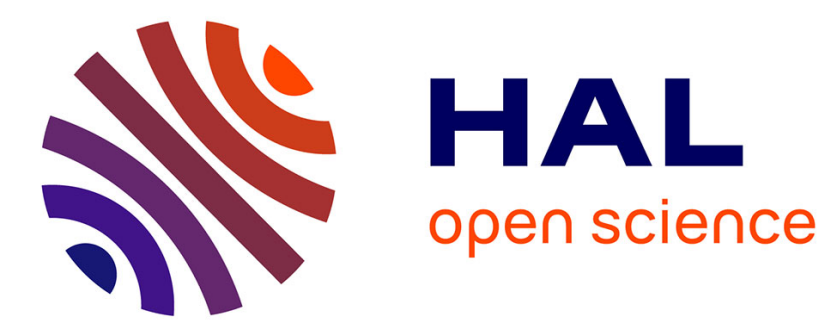

\title{
Evolution structurale d'alliages Pd-Si amorphes en fonction de la température
}

\author{
J.F. Sadoc, M. Laridjani
}

\section{To cite this version:}

J.F. Sadoc, M. Laridjani. Evolution structurale d'alliages Pd-Si amorphes en fonction de la température. Journal de Physique Lettres, 1981, 42 (22), pp.485-489. 10.1051/jphyslet:019810042022048500 . jpa-00231983

\section{HAL Id: jpa-00231983 https://hal.science/jpa-00231983}

Submitted on 1 Jan 1981

HAL is a multi-disciplinary open access archive for the deposit and dissemination of scientific research documents, whether they are published or not. The documents may come from teaching and research institutions in France or abroad, or from public or private research centers.
L'archive ouverte pluridisciplinaire HAL, est destinée au dépôt et à la diffusion de documents scientifiques de niveau recherche, publiés ou non, émanant des établissements d'enseignement et de recherche français ou étrangers, des laboratoires publics ou privés. 


\title{
Evolution structurale d'alliages Pd-Si amorphes en fonction de la température $\left({ }^{*}\right)$
}

\author{
J. F. Sadoc $(* *)$ et M. Laridjani (***) \\ (**) Laboratoire de Physique des Solides, Bât. 510, Université de Paris-Sud, 91405 Orsay, France \\ $\left({ }^{* * *}\right)$ CEN Saclay, DPC/SSS, 91191 Gif sur Yvette Cedex, France
}

(Reçu le 3 juillet 1981, accepté le 2 octobre 1981)

\begin{abstract}
Résumé. - Nous avons étudié sur deux types d'alliages $\mathrm{Pd}_{80}-\mathrm{Si}_{20}$ préparés par pulvérisation cathodique et par trempe ultra-rapide l'influence de la température sur la structure.

Les fonctions d'interférence ont été obtenues par la méthode de diffraction des rayons $\mathrm{X}$ avec analyse d'énergie à angle fixe (méthode non dispersive). Cette méthode permet l'étude de la diffraction d'un échantillon maintenu dans un four à température bien déterminée.

Nous n'observons pas de différence fondamentale dans l'évolution des deux types d'échantillon. Les fonctions d'interférence $I(K)$ et de distribution radiales $W(R)$ évoluent avec un accroissement de la température $\left(20 \rightarrow 340^{\circ} \mathrm{C}\right)$. Par contre aucune évolution sensible des fonctions $I(K)$ et $W(R)$ à $300^{\circ} \mathrm{C}$ n'est observée lors d'un maintien $24 \mathrm{~h}$ à cette température. Si la durée de maintien au-delà de $300^{\circ} \mathrm{C}$ est courte (quelques heures), on observe un comportement quasi-réversible. Par contre, si le maintien à haute température $\left(\sim 300^{\circ} \mathrm{C}\right)$ est long $(\sim 24 \mathrm{~h})$, les fonctions $I(K)$ et $W(R)$ obtenues après retour à $20^{\circ} \mathrm{C}$ ont évolué par rapport aux fonctions initiales. Ces résultats peuvent être interprétés par l'accroissement de la symétrie locale (sites tétraédriques plus parfaits) avec l'accroissement de la température.
\end{abstract}

\begin{abstract}
We have studied the thermal behaviour of two kinds of amorphous $\mathrm{Pd}_{80} \mathrm{Si}_{20}$ alloys prepared by sputtering and by rapid quenching from the melt.

Interference functions were determined by the X-ray non-dispersive method. This method allows the diffraction study of a sample held in a furnace at a stable temperature. We do not observe any difference between the two kinds of samples.

Interference and radial distribution functions evolve with increasing temperature $\left(20^{\circ} \rightarrow 340^{\circ} \mathrm{C}\right)$. Nevertheless no sensitive evolution of the functions $I(K)$ and $W(R)$ at $300^{\circ} \mathrm{C}$ is observed during an isothermal annealing of $24 \mathrm{~h}$ If the isothermal annealing at high temperature $\left(\sim 300^{\circ} \mathrm{C}\right)$ is short, there is a quasi-reversible behaviour; but if the annealing is long $(24 \mathrm{~h})$ the functions $I(K)$ and $W(R)$ obtained after return to $20^{\circ} \mathrm{C}$ are modified relative to the initial functions. These results are analysed in terms of an increase of the tetrahedral perfection.
\end{abstract}

La stabilité des composés amorphes est encore un phénomène mal compris. Il est admis que l'état cristallin est l'état le plus stable, l'énergie interne étant minimum. Par contre, il n'est pas clairement montré si l'état amorphe correspond à un équilibre métastable ou à un état hors d'équilibre avec une évolution extrêmement lente vers l'état cristallin.

Les expériences que nous présentons ici ne répon-

$\left({ }^{*}\right)$ Cet article a été présenté au Congrès de la Société Française de Physique (Clermont-Ferrand, juillet 1981) dans le cadre du Colloque « Verres et Amorphes ».

This article was presented at the Meeting of the French Physical Society (Clermont-Ferrand, July 1981) at the Symposium on « Glass and Amorphous Materials ». dent pas directement à cette question, mais contribueront peut-être à élucider ce problème difficile.

Les échantillons de $\mathrm{Pd}_{80} \mathrm{Si}_{20}$ ont été préparés soit par trempe ultra-rapide à partir du liquide, soit par pulvérisation cathodique.

1. Principe de l'expérience. - Les échantillons ont été étudiés par diffraction de rayon $\mathrm{X}$ à température stabilisée dans un four à atmosphère contrôlée (vide primaire ou hélium gazeux). De façon à réduire autant que possible les temps de mesure et à accroître la reproductibilité des résultats, nous avons utilisé la méthode de diffraction non dispersive avec analyse d'énergie. Pour cela tout le spectre d'émission d'un tube de rayon $\mathrm{X}$ (anticathode d'argent) est analysé 
après diffraction par l'échantillon à l'aide d'un détecteur solide $\mathrm{Si}(\mathrm{Li})$ [1].

Dans la relation $K=4 \pi \sin \theta / \lambda$ (ou bien

$$
K=4 \pi \sin \theta . E / 12400),
$$

on fait varier $\lambda$ (ou $E$ en $\mathrm{eV}$ ) de façon à explorer un domaine de l'espace réciproque, alors que dans une expérience classique de diffraction c'est $\theta$ que l'on explore. Trois points importants ont orienté notre choix vers cette méthode :

- La géométrie du montage est très simplifiée car l'absence de balayage en angle permet l'utilisation d'un four enveloppant bien l'échantillon.

- Les temps d'acquisition d'un spectre sont courts (500 à $1000 \mathrm{~s})$.

- L'absence de pièce mobile, donc l'invariabilité des paramètres géométriques durant un cycle complet d'expériences est un facteur contribuant à avoir une précision relative, sur la variation des fonctions d'interférence très supérieure à la précision absolue sur ces fonctions qui dépendent de paramètres pas très bien déterminés (absorption, spectre du tube de rayon $\mathrm{X}$, facteur de diffusion, etc.).

Les taux de comptage utilisés conduisent à une précision relative sur les spectres de l'ordre de $1 \%$. La stabilité des conditions expérimentales permet alors d'apprécier des variations des détails des fonctions d'interférence avec une précision du même ordre. D'autre part, les résultats que nous présentons ont été répétés plusieurs fois sur des échantillons nouveaux afin de confirmer les faibles variations des détails des fonctions d'interférence.

L'ensemble des fonctions présentées a été obtenu par recoupement de résultats obtenus pour trois angles de mesure $\left(2 \theta=25,40\right.$ et $\left.70^{\circ}\right)$ et un domaine d'énergie des rayons $\mathrm{X}$ variant de 6,6 à $20 \mathrm{keV}$. Une fonction de référence a été déterminée par diffraction angulaire classique à l'aide d'un goniomètre $(\theta-2 \theta)$ à la température ambiante (Fig. 1). La longueur d'onde utilisée

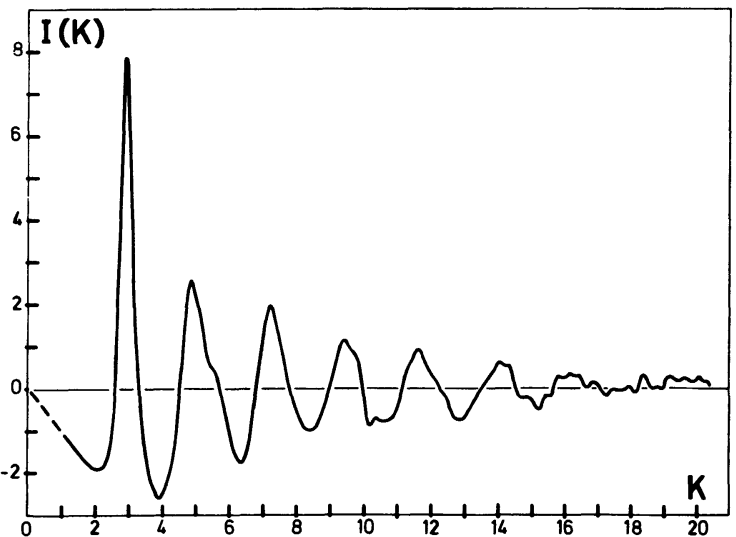

Fig. 1. - Fonction d'interférence de $\mathrm{Pd}_{80} \mathrm{Si}_{20}$ obtenue par diffraction des rayons $\mathrm{X}$ (méthode de dispersion angulaire).

[A $\mathrm{Pd}_{\mathbf{8 0}} \mathrm{Si}_{20}$ interference function obtained by X-ray diffraction (angular dispersion).] $(\lambda=0,56 \AA ; \operatorname{AgK} \alpha)$ a permis d'obtenir une fonction jusqu'à $K=20,4 \AA^{-1}$. Une fonction de distribution radiale a été déduite par transformation de Fourier (Fig. 2). Les deux fonctions désormais classiques sont en accord avec les résultats antérieurs [2,3]. Néanmoins, il est exceptionnel que par diffraction des rayons X une valeur de $K_{\max }=20 \AA^{-1}$ soit atteinte. Cette fonction a permis l'étalonnage des fonctions obtenues par la méthode non dispersive en comparant les spectres obtenus à la température ambiante avec la courbe de référence. Les corrections ainsi calculées ont ensuite été appliquées aux spectres mesurés (Fig. 3) à diverses températures de façon à obtenir des fonctions d'interférence normalisées.

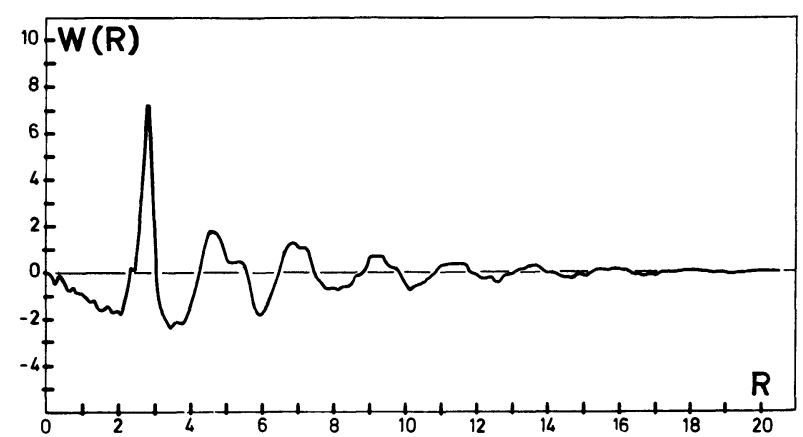

Fig. 2. - Fonction de distribution radiale réduite, obtenue par transformation de Fourier de la fonction d'interférence avec $K_{\mathrm{M}}=20,4 \AA^{-1}$.

[Radial distribution function obtained by Fourier transform from interference function with $K_{\mathrm{M}}=20.4 \AA^{-1}$ ]

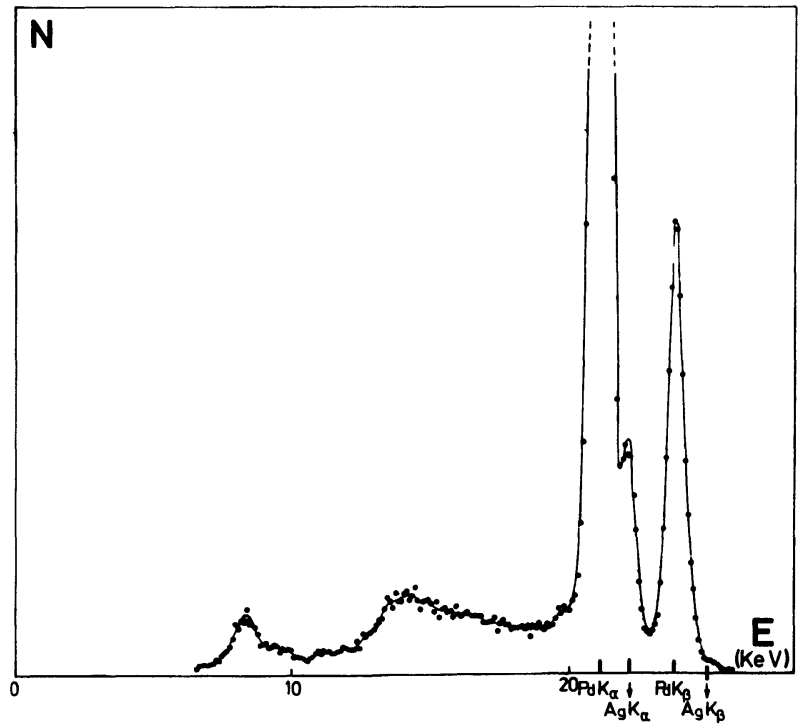

Fig. 3. - Exemple de spectre expérimental. On distingue les raies caractéristiques du tube $\mathrm{AgK} \alpha$ et $\mathrm{K} \beta$, les raies de fluorescence du palladium $(K \alpha$ et $K \beta)$. On remarque la modulation du spectre continu par les anneaux d'interférence.

[An example of experimental spectrum. Characteristic lines of the target $(\mathrm{AgK} \alpha$ and $\mathrm{K} \beta)$ and fluorescence line $(\operatorname{PdK} \alpha$ and $\mathrm{K} \beta)$ are shown. Note the modulation of the continuous spectrum by interference rings.] 
De ces fonctions, une transformation de Fourier (limite d'intégration $K_{\max }=12,5 \AA^{-1}$ ) donne des fonctions de distribution radiale. Afin d'accroître la résolution de ces fonctions, nous les avons prolongées entre $12,5 \AA^{-1}$ et $20,4 \AA^{-1}$ en utilisant la queue de la fonction de référence. Ceci revient à négliger les effets thermiques sur cette partie de courbe.

2. Résultats. - 2.1 CRistallisation. - Les premières expériences ont consisté à suivre des montées en température à vitesse de chauffe régulière (avec des paliers de $500 \mathrm{~s}$ pour les comptages) depuis $20^{\circ} \mathrm{C}$ jusqu'à $400^{\circ} \mathrm{C}$ en $4 \mathrm{~h}$ environ. L'apparition de la cristallisation est très brutale à $370^{\circ} \mathrm{C}$.

Nous avons également étudié l'effet de longs maintiens à $200^{\circ} \mathrm{C}$ pendant 6 jours ; aucune cristallisation n'apparaît. Il en est de même quand la température reste $24 \mathrm{~h}$ à $300^{\circ} \mathrm{C}$.

2.2 EVOLUTION STRUCTURALE. - La comparaison entre échantillons trempés et pulvérisés n'indique pas de différences notables dans l'allure et le comportement des fonctions d'interférence. Dès $200^{\circ} \mathrm{C}$, on remarque une évolution des fonctions d'interférence,
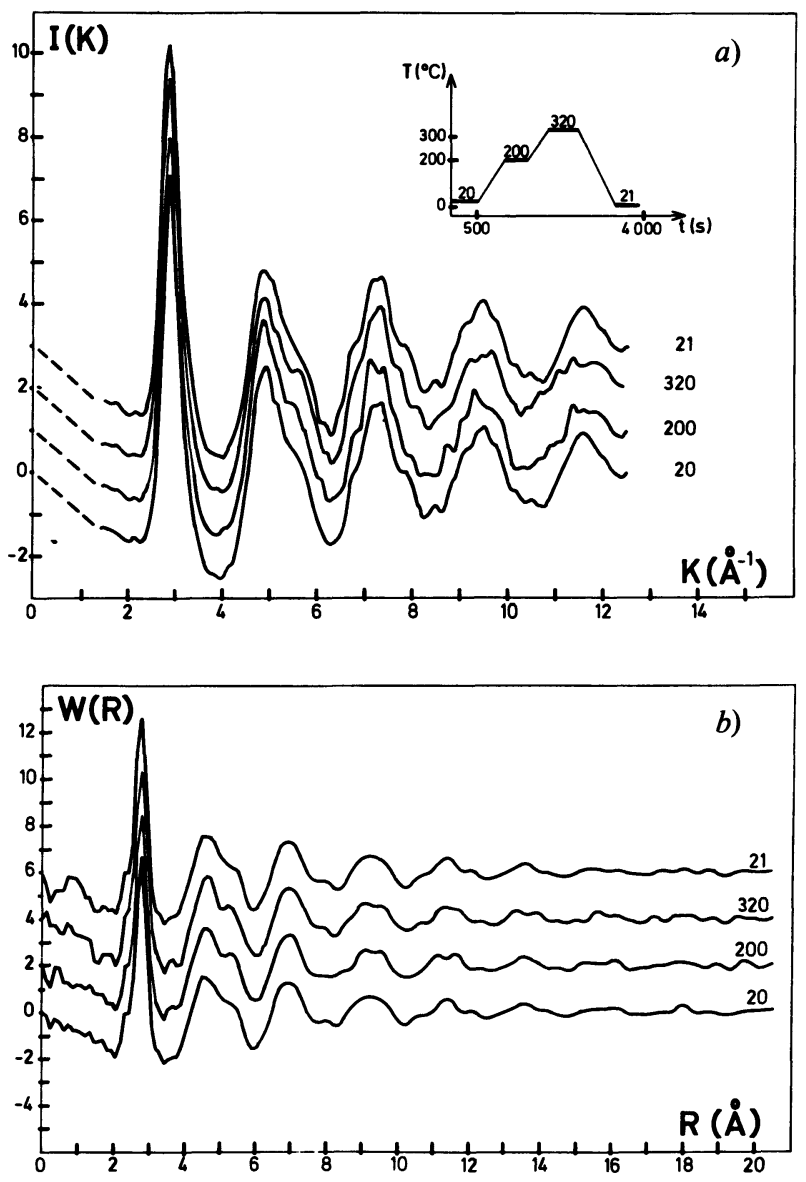

Fig. 4. - Evolution de $I(K)$ et de $W(R)$ lors d'un cycle court $\left(20^{\circ} \mathrm{C}, 340^{\circ} \mathrm{C}, 20^{\circ} \mathrm{C}\right)$.

[Some evolution of $I(K)$ and $W(R)$ during a short cycle $\left(20^{\circ} \mathrm{C}\right.$, $\left.340^{\circ} \mathrm{C}, 20^{\circ} \mathrm{C}\right)$.] en particulier un renforcement de l'épaulement du deuxième anneau. Par contre, aucune variation de l'intensité du premier anneau n'est observable. Cette évolution continue jusqu'à $340^{\circ} \mathrm{C}$ (Fig. 4).

Si le maintien au-delà de $250^{\circ} \mathrm{C}$ ne dépasse pas 3 à $4 \mathrm{~h}$ lors du retour à la température ambiante, la fonction d'interférence reprend son allure initiale. Nous avons alors étudié l'évolution lorsque la température est stabilisée à environ $300^{\circ} \mathrm{C}$. On n'observe pas de modification dépendant du temps car les courbes obtenues à $300^{\circ} \mathrm{C}$ au bout d'une heure ou de $24 \mathrm{~h}$ sont semblables avec, en particulier, un épaulement sur le $2^{\mathrm{e}}$ anneau, assez marqué. Mais la fonction d'interférence obtenue après retour à la température ambiante a subi des modifications relativement à la courbe initiale obtenue à $20^{\circ} \mathrm{C}$. Elle conserve un souvenir de l'allure de la fonction à $300^{\circ} \mathrm{C}$.

Ces modifications sont surtout observables sur l'épaulement du $2^{e}$ anneau de la fonction d'interférence (Fig. 5a). En particulier, la comparaison des courbes des figures $4 a(21)$ avec les courbes figure $5 a(22)$ et figure $4 b(21)$ et figure $5 b(22)$ permet d'apprécier les variations des fonctions étudiées.

Nous observons donc une quasi-réversibilité lors de cycles thermiques courts $(\sim 1 \mathrm{~h})$ entre la température
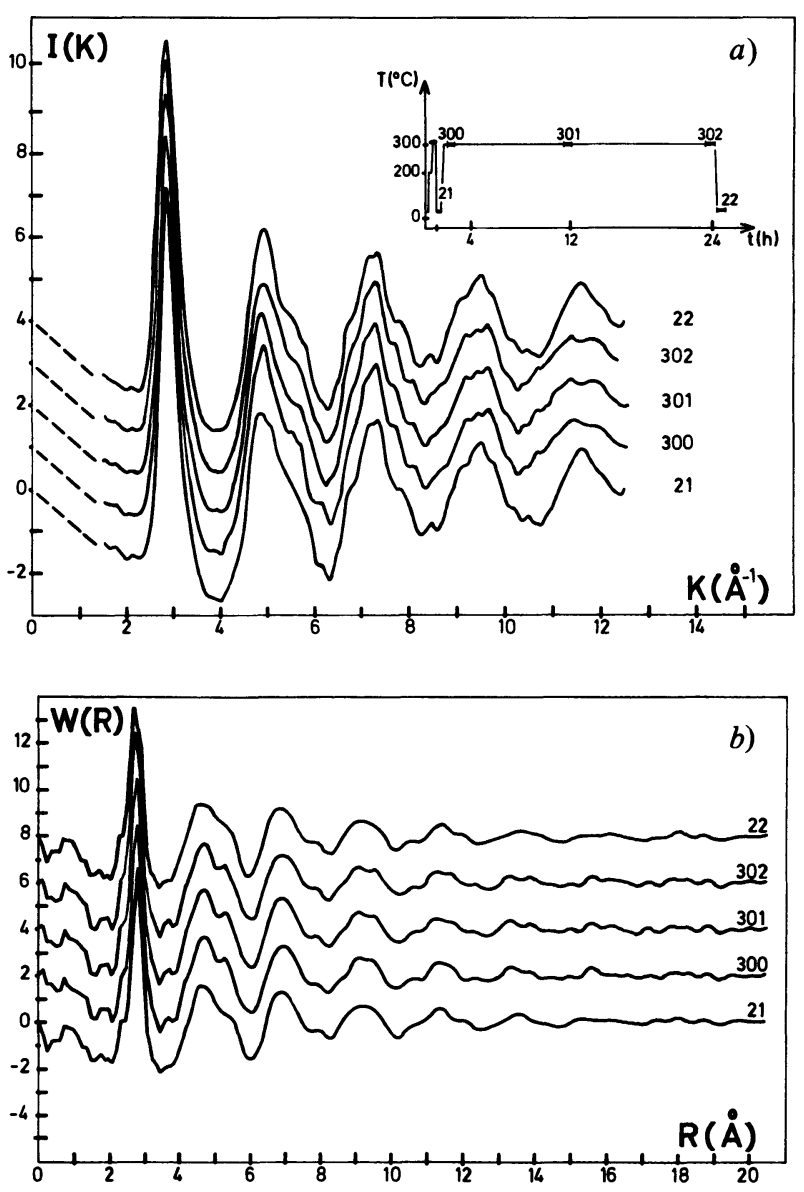

Fig. 5. - Effet d'un maintien prolongé à $300^{\circ} \mathrm{C}$ sur $I(K)$ et $W(R)$.

[Influence of a long annealing at $300^{\circ} \mathrm{C}$ on the function $I(K)$ and $W(R)$.] 
ambiante et une température juste inférieure à la température de cristallisation. Nous observons également un effet de réorganisation pour des durées de recuit à $300{ }^{\circ} \mathrm{C}$ de l'ordre de $24 \mathrm{~h}$. Notons enfin un léger effet sur le pied du $1^{\mathrm{er}}$ anneau de la fonction d'interférence $\left(K \sim 3,5 \AA^{-1}\right)$ : la fonction décroît moins rapidement à basse température qu'à haute température. Quoique à la limite de la précision expérimentale, cet effet semble moins marqué pour les échantillons pulvérisés.

3. Discussion. - L'évolution des fonctions réduites de distribution radiale est caractérisée par un accroissement de la symétrie de l'ordre local moyen avec la température. Ceci peut être observé sur plusieurs des distances caractéristiques des fonctions de distribution radialé:-Des travaux antérieurs [4] permettent d'expliquer l'allure du début des fonctions de distribution radíale $(R<6 \AA)$ par deux familles de distances :

- distances Pd-Pd :

$11^{\mathrm{er}}$ voisins à $\sigma \simeq 2,80 \AA$,

$2^{\mathrm{e}}$ voisins à $4,7 \AA=1,80 \sigma$,

$3^{\mathrm{e}}$ voisins à $5,4 \AA \approx 2 \sigma$;

- distances Pd-Si :

$1^{\mathrm{er}}$ voisins $\sigma^{\prime}=2,40 \AA$,

$2^{\mathrm{e}}$ voisins à $4,40 \AA$,

$3^{\mathrm{e}}$ voisins à $5,12 \AA \approx \sigma+\sigma^{\prime}$

les distances $\mathrm{Si}-\mathrm{Si}$ très peu visibles aux rayons $\mathrm{X}$ ayant un effet insignifiant.

Sur les fonctions obtenues à la température ambiante on distingue quelques détails caractéristiques de ces distances (Fig. 2) :

- les deux distances de premiers voisins Pd-Pd et $\mathrm{Pd}-\mathrm{Si}$ sont presque complètement résolues sur les courbes obtenues par coupure à $K=20 \AA^{-1}$,

- les distances de troisièmes voisins apparaissent comme un épaulement sur le flanc du deuxième anneau. De faibles changements de pente sur cet anneau peuvent être attribués aux deux familles $\mathrm{Pd}-\mathrm{Pd}$ et $\mathrm{Pd}-\mathrm{Si}$.

Lors du chauffage, on observe une symétrisation des pics principaux correspondant aux distances Pd-Pd et une diminution des pics $\mathrm{Pd}-\mathrm{Si}$. Ceci est très net pour la première distance. Donc, les distances $\mathrm{Pd}-\mathrm{Si}$ se rapprochent des distances $\mathrm{Pd}-\mathrm{Pd}$. On n'observe pas de variation de la largeur du $1^{\mathrm{er}}$ pic, probablement en raison de l'élargissement résultant de l'effet de terminaison.

Nous concluons donc à un accroissement de la symétrie locale moyenne lors de l'élévation de la température.

Dans des travaux antérieurs, la comparaison des résultats de diffraction des rayons $\mathrm{X}$ et des neutrons a permis d'obtenir des fonctions d'interférence partielles pour $\mathrm{Pd}-\mathrm{Pd}$ et $\mathrm{Pd}-\mathrm{Si}$ avec une précision d'environ $5 \%$ à $10 \%$ dans un domaine permettant d'obtenir par transformation de Fourier des fonctions de distribution partielles (Fig. 6).

Sur ces fonctions partielles on remarque que le pic des premiers voisins Pd-Si est beaucoup plus dissy-

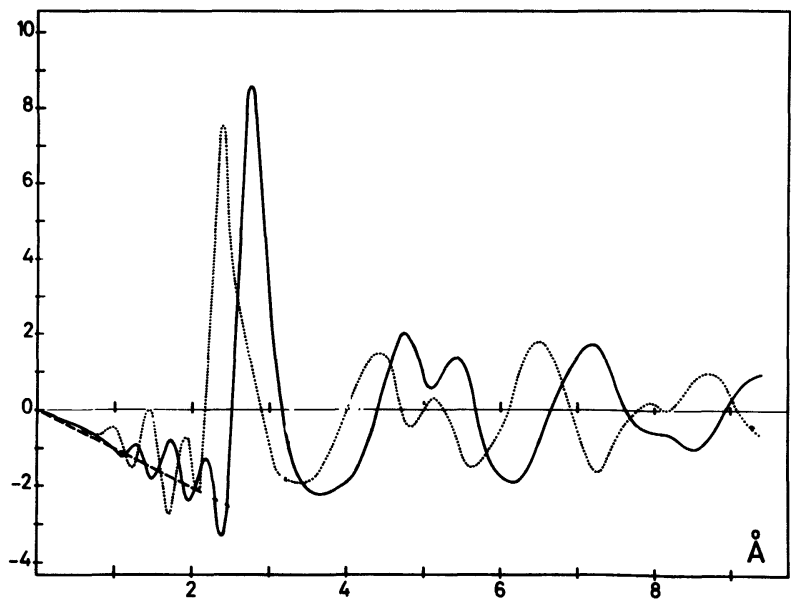

Fig. 6. - Fonctions partielles. $-W_{\mathbf{P d}-\mathbf{P d}} ; \ldots . . W_{\mathbf{P d}-\mathrm{Si}}$.

[Partial functions. $\left.-W_{\mathbf{P d}-\mathrm{Pd}} ; \ldots . . W_{\mathbf{P d}-\mathrm{Si}}.\right]$

métrique que celui des distances Pd-Pd. Donc la partie répulsive du potentiel d'interaction $\mathrm{Pd}-\mathrm{Si}$ est beaucoup plus abrupte que celle du potentiel Pd-Pd; par conséquent, une élévation de température accroît la distance $\mathrm{Pd}-\mathrm{Si}$ moyenne et symétrise la répartition. $\mathrm{Si}$ le maintien à haute température est court, cet effet disparaît par retour à la température ambiante. Par contre, si le maintien à environ $300^{\circ} \mathrm{C}$ est long $(24 \mathrm{~h})$ lors du retour à la température ambiante, nous voyons décroître la distance Pd-Si entre premiers voisins, mais nous observons une modification de la répartition des distances de $2^{e}$ et $3^{e}$ voisins. Ceci apparaît également comme un accroissement de l'épaule sur le $2^{\mathrm{e}}$ anneau de la fonction d'interférence. Une augmentation de la perfection des sites tétraédriques (Fig. 7) ayant ces effets, c'est l'hypothèse que nous adopterons. Un recuit a donc tendance à renforcer la symétrie des sites tétraédriques, c'est-à-dire à accroître l'ordre local. L'étude

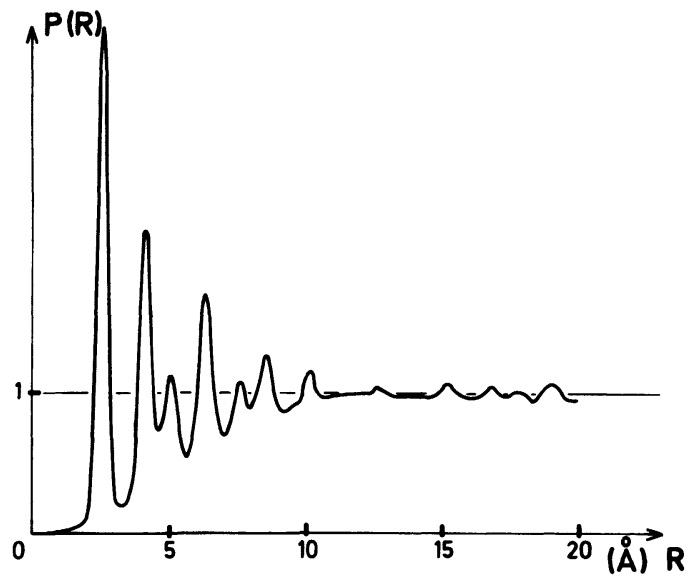

Fig. 7. - Fonction de distribution radiale pour un modèle d'empilement compact tétraédrique avec une grande perfection des tétraèdres.

[Radial distribution function for close packing model with a high tetrahedral perfection.] 
des structures amorphes montre que ceci entraîne un accroissement des défauts de la structure [5], donc des fluctuations de la densité plus importantes. Une mise en ordre de ces défauts n'est d'ailleurs pas à exclure comme le montrent de récentes expériences de diffusion centrale [6].

\section{Bibliographie}

[1] Gasgnier, M. and Malaurent, J. C., J. Appl. Crystallogr. 11 (1978) 141.

[2] Suzuki, K., Fukunaga, T., Misawa, M. and Masumoto, T., Mat. Sci. Eng. 23 (1976) 215.

[3] Andonov, P., Comparaison amorphe-liquide PdSi, J. NonCryst. Solids 22 (1976) 145.

[4] SADoc, J. F., Thesis, Université de Paris XI, Orsay (1974).

[5] SAdoc, J. F., J. Non-Cryst. Solids 44 (1981) 1.

[6] Flank, A. M., Laridjani, M. et Naudon, A., C.R. Hebd. Séan. Acad. Sci., Paris (1981) 288. 